\title{
Combined Anesthesia for Laparoscopic Nissen Fundoplication and Surgical Gastrostomy in a child with Krabbe's disease
}

\author{
E. Carvalho ${ }^{1}$, P. Almeida ${ }^{1}$, S. Alves ${ }^{1}$, V. Antunes ${ }^{1}$, M. Gonçalves ${ }^{1}$. \\ ${ }^{1}$ Hospital de Braga, Department of Anesthesiology, Braga, Portugal.
}

\section{Background:}

Krabbe disease is a severe neurological condition. Initial signs and symptoms typically include irritability, muscle weakness, feeding difficulties, stiff posture and delayed mental and physical development. As the disease progresses, muscles continue to weaken, affecting the infant's ability to move, chew, swallow, and breathe ${ }^{1}$. Of concern to the anesthetist is the high prevalence of seizure disorders, gastroesophageal reflux with the risk of aspiration and airway complications related to poor pharyngeal muscle control and copious oral secretions ${ }^{2}$.

\section{Case Report:}
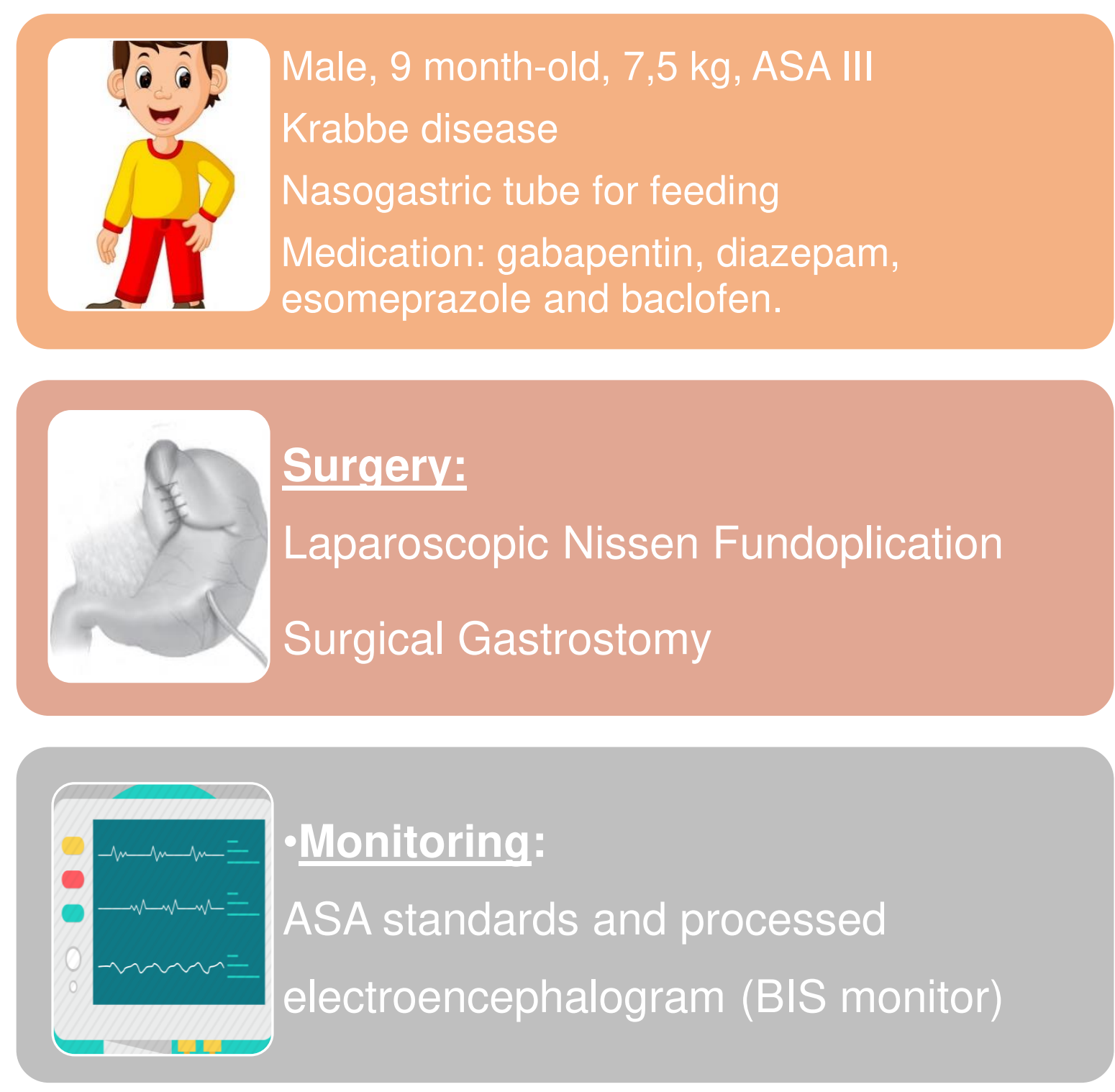

\section{Anesthesia:}

- Patient was sedated with IV midazolam (1 mg).

- In left lateral decubitus, we placed an epidural catheter at T7T8 level under ultrasound control via caudal approach (Fig 1).

- Inhaled induction with sevoflurane was performed after epidural administration of $3 \mathrm{mg}$ of ropivacaine $(0.2 \%)$.

- 20 minutes after induction, surgical incision was performed.

- General anesthesia was maintained with sevoflurane and a mixture of oxygen and air through a facial mask and keeping spontaneous ventilation.

\section{Results:}

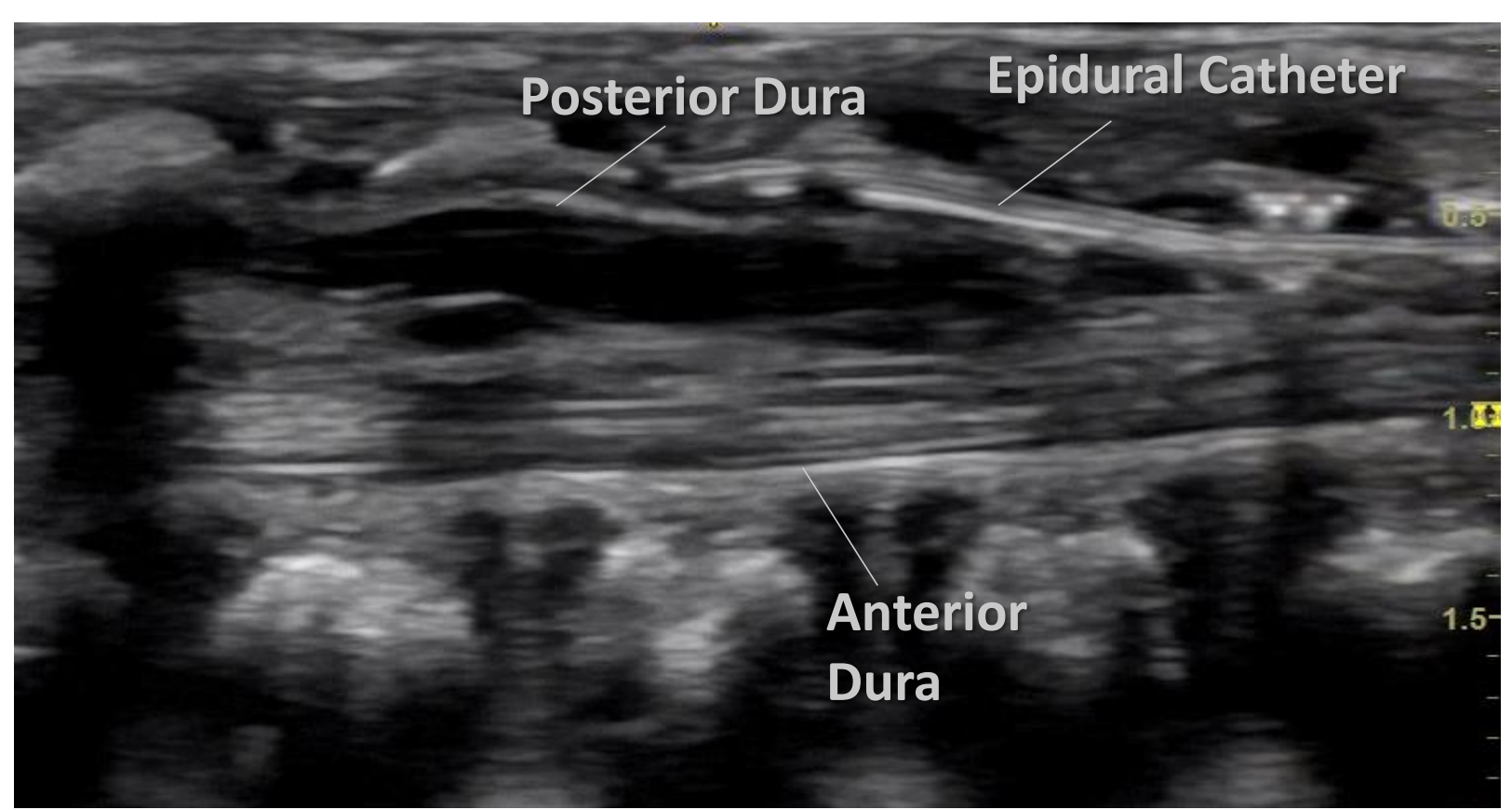

Fig. 1: Ultrasound image of epidural catheter path

\section{Perioperative:}

- Multimodal analgesia was provided with administration of IV paracetamol (120 mg) and IV ketorolac (4 mg).

- Patient remained hemodynamically stable throughout the procedure.

- Surgery lasted 2 hours and was uneventfully.

\section{Postoperative:}

- The patient was transferred for Pediatric Intensive Care Unit (PICU) for postoperative vigilance.

- Analgesic protocol: bolus of ropivacaine $0.1 \%$ (3 $\mathrm{mg})$ was given every 4 hours for 24 hours + paracetamol 120 mg every 8 hours and ketorolac $4 \mathrm{mg}$ every 12 hours until discharge. The patient remained comfortable with no need of opioid rescue.

- Discharge from the hospital occurs 72 hours after surgery without complications

\section{Conclusions:}

General anesthesia with spontaneous ventilation combined with epidural anesthesia is an alternative in laparoscopic Nissen fundoplication avoiding the need of orotracheal intubation and avoiding the airway complications related to poor pharyngeal muscle control. 